Reliability, uncertainty and costs in the evolution of animal learning

\begin{abstract}
Aimee S. Dunlap ${ }^{a} \&$ David W. Stephens ${ }^{b}$
${ }^{a}$ Department of Biology, University of Missouri, St. Louis

One University Blvd, St. Louis, MO 63121, USA

aimee.dunlap@umsl.edu

${ }^{\mathrm{b}}$ Department of Ecology, Evolution, \& Behavior, University of Minnesota, Twin Cities 1987 Upper Buford Circle, St. Paul, MN 55108, USA

steph031@umn.edu
\end{abstract}




\begin{abstract}
Learning is a fundamental mechanism in the behavior of animals. Theorists have long proposed that learning is an adaptation to environmental change, but change itself can present a logical paradox in that change can select both for and against learning. One way to resolve this paradox is through separating change into different components, such as the reliability of stimuli that can be used as cues for behavior, and the certainty with which those cues predict the best behavior to employ. Simple models using these components of change can be successfully applied through experimental evolution to directly test hypotheses of when learning and innate preference will each be favored evolutionarily, as well as when prepared learning will evolve. Costs of learning and, in particular, economic costs such as opportunity costs, should influence when learning will be favored. Although experimental evolution of learning can be difficult in practice, the benefits of the approach far outweigh difficulties. Future studies might approach the role of opportunity costs and how reliability and certainty fully interact to influence the evolution of learning.
\end{abstract}


A songbird learns the distinctive call of its neighbor, a rat learns how to extract the seeds from a pine cone, a vervet learns that some individuals signal more reliably than others and so on. Surely, every serious student of behavior recognizes the importance and pervasiveness of learning. Yet, many behavioral ecologists see learning as outside of their field. Learning, the thinking goes, is a topic for psychologists while evolution and ecology are the central focus of behavioral ecology. The problem with this is that learning, like any other behavioral trait, needs to be understood in terms of the ecological context in which it functions, and the evolutionary forces that have shaped it. This article considers the evolutionary forces that have shaped learning, and in doing so it shows how the behavioral ecology of learning is emerging as a significant field within our discipline.

A long-standing argument holds that learning is an adaptation to environmental change, e.g. [1-8]. The logic behind this flows from the following observation: Suppose that a species evolves in the same unchanging environment over many generations. Clearly, there is no reason for our hypothetical animal to change its behavior, and hence no reason to attend to experience to guide this change. The argument from absolute fixity tells us that we need some change to promote learning, it does not necessarily mean that more change promotes learning more strongly. In fact, this is not true. One could instead imagine a completely random world in which the conditions today bear no predictable relationship to tomorrow. That's a lot of change, but it's clear that learning cannot pay in this case because experience today tells the animal nothing about conditions tomorrow. This can be called the 'paradox of variability' because it suggests that the variability can both favor and disfavor learning. Moreover, it suggests that something interesting may be happening in the space between absolutely fixity and complete randomness. 
Solving the paradox of variability

Figure 1 shows one way to resolve the paradox of variability. The figure shows the results of a model that considers two components of environmental variation that have opposite effects on the fitness value of learning [9]. Certainty broadly represents the animal's ability to choose an appropriate action without further experience. Clearly, when the condition of the environment changes frequently and randomly there will be low certainty, or equivalently high uncertainty. High uncertainty favors learning, just as in the traditional "learning is an adaptation to change" argument. Reliability expresses what experience tells the animal about the best action, specifically, the conditional probability that a given experience predicts this best action. If experience is perfectly reliable then each possible experience correlates perfectly with the best action in the current environmental situation. High reliability favors learning, and any change that disrupts the correlation between experience and action will reduce the value of learning. Moreover, the model of figure 1 suggests that these two variables combine in a proportional way. When prior certainty is high - implying that the animal can choose an appropriate action with reasonable accuracy - then reliability must also be high to make learning worthwhile. Notably, the model predicts that neither learning nor non-learning are favored in a fixed environment $(p=1, q=1)$, because learning and non-learning have the same fitness in this condition.

While there are many ways to model the fitness value of learning and resolve the paradox of variability e.g. $[2,10]$, we focus on this simple model because it has been useful experimentally. The central idea behind all models of learning evolution is that statistical properties of the selective environment have favored or disfavored learning and plasticity during the course of 
evolution. A central problem in evaluating these models is that we do not have measures of properties like uncertainty and reliability in evolutionarily relevant time periods. This presents a fundamental problem of evolutionary time, where rigorous tests of these historical variables in the evolution of learning have not previously been easily tractably testable.

Recent advances in the techniques of experimental evolution suggest a way forward that solves the evolutionary time problem. The idea here is that one can create a range of selective environments, say that vary in the uncertainty and reliability, and expose replicate populations of animals to these environments for many generations. Mery and Kawecki's pioneering work provided a technical break-through in the study of learning evolution by developing an experimental procedure that allowed investigators to manipulate statistical properties of the selective environment over many generations $[11,12]$, and testing the idea that change favors learning and fixity favors non-learning. Figure 2 gives an overview of these procedures. Dunlap and Stephens [9] expanded this new experimental system to test replicate populations of Drosophila in the two most extreme cases of figure 1-low certainty and high reliability, and high certainty and low reliability. Figure 3 shows their results; flies in the low certainty/high reliability treatments did show improved learning abilities after 30 generations of experimental evolution. When experience is unreliable, however, learning ability does not decline compared to controls.

\section{Prepared learning}

Psychologists originally thought of learning as a general purpose association-forming mechanism, but in the 1960s evidence began to appear that showed that some kinds of 
association where formed more readily than others. The most famous example is the study of Garcia and Koelling [13] which showed that rats quickly learn to avoid bad tasting water, but had difficulty learning to avoid a water source associated with sounds and lights. Following this ground breaking work, a large number of studies have shown that animals seem better prepared to learn some types of associations e.g. [14-17]. Nearly everyone agrees that the explanation of these differences in learning abilities must flow from evolutionary history, but until recently this has been justifiably criticized as a just-so story. We face two problems in overcoming this just-so story critique. First we must have a specific hypothesis about the aspects of evolutionary history that favor prepared learning, and second we must overcome the evolutionary time problem again. As explained above, experimental evolution gives us a way to overcome the evolutionary time problem, so we focus on formulating a testable hypothesis.

In the problem of prepared learning we consider two types of experience (a taste or flashing lights), and we ask whether it pays to attend to one type of experience over the other. The model of figure 1 asked a similar question: should you act on the basis of what you already know or should you use experience to guide your actions? Now imagine that two types of experience are possible, S (say an odor difference) and $\mathrm{T}$ (say a color difference). We can characterize the accuracy derived from following $\mathrm{S}$ by the parameter $q_{S}($ read the reliability of $\mathrm{S})$; similarly we characterize the accuracy derived from following $\mathrm{T}$ by the parameter $q_{T}$. This suggests a simple hypothesis: follow the experience with the highest reliability, or follow $\mathrm{S}$ if $q_{S}>q_{T}$. We would predict therefore a lineage that experience selection of many generations where $q_{S}$ is high and $q_{T}$ is low would show prepared learning for $\mathrm{S}$ (odor in our example) but ignore differences in T (e.g color), and vice-versa. 
To test this hypothesis Dunlap and Stephens modified the oviposition preference task (Figure 2), to create oviposition substrates that varied in both odor and color [18]. As with their original study, flies experienced the aversive chemical quinine on one of the two oviposition substrates in the first presentation, and then experienced the two media types - now varying in color and odor-in a second phase without quinine. Offspring were raised from only one of the plates of media, so the decision about where to lay eggs had severe fitness consequences. What varied in this study was the predictive power of the quinine-color vs. the quinine-odor association. The strongest predictions are in the conditions where one type of experience has high reliability and the other has low reliability. Figure 4 shows the results of learning assays on these two critical experimental groups after 40 generations of experimental evolution. Consistent with our hypothesis of prepared learning, flies selectively learn patterns that have provided reliable indications of the appropriate actions during their selective history.

The Dunlap \& Stephens prepared learning experiment tests the claim that selection should favor attending to the single best source of information. However animals are arguably inundated with many potential sources of information. One can reasonably ask why it doesn't pay to integrate multiple sources of information. Recent theoretical work [19] (summarized in figure 4) suggests that the benefit of integrating two types of experience depends on the underlying uncertainty about action---the $p$ parameter of figure 1 . If there is high uncertainty $(p=0.5$ in figure 1), as in the Dunlap and Stephens experiment, then the 'single best' rule applies. If, however, the system is biased in favor of one outcome over the other, combinations of experience can be important. Specifically, combinations can be influential when they confirm the occurrence of a rare event. Suppose for example that it's best to lay eggs on the 'orange flavored' media $80 \%$ of the time; and that two types of 'marks' exist to indicate this - a color mark and an 
odor mark. Theory predicts that a rule of the form "lay eggs on orange, except when both color and odor indicate that pineapple is better" can be better than relying on the single most reliable mark in some situations.

\section{Costs of Learning}

After a long period of mostly conjecture and theory, work on how costs of learning affect the evolution of learning are beginning to take better shape. Primarily the work comes in two forms (trade-offs and molecular costs), and we argue for better inclusion of a third (opportunity costs). First, learning clearly involves trade-offs, particularly in development. Work shows that the extra brain tissue correlating with better learning can trade off with gut tissues [20,21] and reproductive tissue [22], though brain size is arguably not always a predictor of learning ability, e.g. [20,23]. Trade-offs are also seen in life history, including delayed juvenile development [22,24], shorter lifespan [25], and reduced competitive ability [26]. We expect trade-offs of various sorts to interact with change in the environment to determine the adaptive value of learning. A number of clever tests have uncovered trade-offs with energy use, for example showing that immune response and learning can trade off in foraging bees [27-29]. More directly, a high energetic cost of learning can cause measurable cognitive deficits in bees, e.g. [30], and flies facing food shortages disable formation of long-term memory [31], while flies using learning with long-term memory face a quicker death in freezing conditions [32]. On a more molecular level, we assume that learning would come with metabolic costs, in terms of creating and maintaining the synaptic connections underlying the integration, storage, and retrieval of information, e.g. [33]. Models have been developed on the metabolic cost of neural information, e.g. [34], however more recent tests have measured aspects of this cost. For instance 
flies can quickly learn that a food is sweet, to remember that information for very long, the learning experience must come with a metabolic gain $[35,36]$.

The economic costs learning go beyond the expenses of building and maintaining the learning machinery: a learner can pay opportunity costs because the experience required to establish a learned behavior takes time and presumably often results in poor performance while the animal tries and rejects poor alternatives. There are reasons to think that these costs are at least as important as the mechanistic costs of learning, and possibly more important. But while the 'cost of learning' literature has focused on the costs of building and operating the neural machinery of learning, the opportunity costs of learning have received no meaningful empirical attention. The opportunity costs of learning are critical variables in models of learning, e.g. [2], and directly manipulating sampling costs, for instance through changes in reliability, may affect the evolution of learning by increasing neophobia, and limiting the experiences that can inform behavior, e.g. [37]. Our basic framework of reliability and certainty can be extended to include opportunity costs, and a logical result is a stronger role for certainty as opportunity costs increase. Clearly, an important future direction is to develop empirical tools to study the effects of opportunity costs in the evolution of learning, and ultimately to use these tools to contrast the effects of opportunity and mechanistic costs.

\section{Conclusions}

The tension between fixity and change has long been referenced in thinking about what types of environments will favor the evolution of learning, however it is not completely satisfactory in describing change as animals experience it, or how learning is also favored in fixed 
environments. By splitting environmental change into different components, reliability and certainty, this paradox is solved. Through simple modeling we find that reliability and certainty interact to determine when learning should evolve rather than a fixed behavior like an inherited preference. An extension of this model likewise predicts when prepared learning should evolve, based on the reliabilities of observed stimuli. The basic predictions of both models have been tested using experimental evolution studies in flies. Further studies will be required to test the complete interaction of these types of change and how the role of economic costs influence when learning evolves. This is especially the case where alternatives to learning may perform equally as well or better, for instance in new environments [38], under relaxed sexual selection [39], and under direct selection for fixed behavior [40]. Although the learning model sets learning and innate preference as opposing behaviors, we know through innumerable studies that animals can combine inherited preference or bias with acquired experience to determine behavior e.g. [41]. Similarly, inherited bias may both impede and promote the evolution of learning. This interaction across evolutionary time is an important and challenging area of study, as is more broadly, how learning affects evolution writ large [42].

Fundamental theory about the role of environmental change in the evolution of learning remained directly untested for many years. Experimental evolution is a powerful tool in studying the evolution of learning, because it allows us to solve the evolutionary time problem (at least for short-lived species), and experimentally manipulate parameters of environmental change that were previously quite intractable. It is not a simple tool in practice, however: experiments can be very time and labor intensive and potentially risky. The promise of experimental evolution studies lies also in the ability to connect the evolution, ecology, and economics of learning to mechanism. Work on costs and trade-offs for evolved learning has begun this connection, but 
two directions will help to deepen this work. The first is in careful analyses of correlated traits evolving in conjunction with learning in the laboratory, such as exploration style, speed-accuracy in cognition, and risk-reward trade-offs, allowing for very direct tests of hypotheses outlined by Sih \& Del Giudice [43]. The second direction is an expanded approach in studying the genetics of underlying evolved behaviors, e.g. [44-49]. Careful manipulations of different components of environmental change across evolutionary time are integral to making these connections.

\section{Acknowledgements}

The authors acknowledge funding from the US National Science Foundation, NSF IOS-1021183. 
References

1. Stephens DW: On economically tracking a variable environment. Theoretical Population Biology 1987, 32:15-25.

2. Stephens DW: Change, regularity, and value in the evolution of animal learning. Behavioral Ecology 1991, 2:77-89.

3. Papaj DR: Optimizing learning and its effect on evolutionary change in behavior. In Behavioral Mechanisms in Evolutionary Ecology. Edited by Real LA: University of Chicago Press; 1994:133-153.

4. Plotkin HC, Odling-Smee FJ: Learning, change, and evolution: an enquiry into the teleonomy of learning. Advances in the Study of Behavior 1979, 10:1-41.

5. Johnston TD: Selective costs and benefits in the evolution of learning. Advances in the Study of Behavior 1982, 12:65-106.

6. Papaj DR, Prokopy RJ: Ecological and evolutionary aspects of learning in phytophageous insects. Annual Review of Entomology 1989, 34:315-350.

7. Dridi S, Lehmann L: Environmental complexity favors the evolution of learning. Behavioral Ecology 2016, 27:842-850.

8. Aoki K, Feldman MW: Evolution of learning strategies in temporally and spatially variable environments: a review of theory. Theor Popul Biol 2014, 91:3-19.

9. Dunlap AS, Stephens DW: Components of change in the evolution of learning and unlearned preference. Proceedings of the Royal Society of London B 2009, 276:32013208. 
10. Kerr B, Feldman MW: Carving the cognitive niche: Optimal learning strategies in homogeneous and heterogeneous environments. Journal of Theoretical Biology 2003, 220:169-188.

11. Mery F, Kawecki TJ: Experimental evolution of learning ability in fruit flies. Proc Natl Acad Sci U S A 2002, 99:14274-14279.

12. Mery F, Kawecki TJ: The effect of learning in experimental evolution of resource preference in Drosophila melanogaster. Evolution 2004, 58:757-767.

13. Garcia J, Koelling RA: Relation of cue to consequence in avoidance learning. Psychonomic Science 1966, 4:123-124.

14. Domjan M: The Garcia-Koelling selective association effect: a historical and personal perspective. International Journal of Comparative Psychology 2015, 28.

15. Shettleworth SJ: Constraints on learning. Advances in the Study of Behavior 1972, 4:1-68.

16. Seligman MEP: On the generality of the laws of learning. Psychological Review 1970, 77:406-418.

17. Krause MA: Evolutionary perspectives on learning: conceptual and methodological issues in the study of adaptive specializations. Anim Cogn 2015, 18:807-820.

*18. Dunlap AS, Stephens DW: Experimental evolution of prepared learning. Proceedings of the National Academy of Science USA 2014, 111:11750-11755.

The authors report the first model linking reliability to prepared learning and test it using experimental evolution in fruit flies. Flies evolved prepared learning for stimuli reliably linked to fitness across generations. 
*19. Rubi TL, Stephens DW: Does multimodality per se improve receiver performance? An explicit comparison of multimodal versus unimodal complex signals in a learned signal following task. Behavioral Ecology and Sociobiology 2016, 70:409-416.

Using operant techniques in blue jays, the authors test the hypothesis that multimodel signals are widespread because they enhance learning in receivers. While reliablily matters, the jays were able to learn quite well under every condition tested regardless of the modality.

20. Kotrschal A, Rogell B, Bundsen A, Svensson B, Zajitschek S, Brannstrom I, Immler S, Maklakov AA, Kolm N: The benefit of evolving a larger brain: big-brained guppies perform better in a cognitive task. Anim Behav 2013, 86:e4-e6.

21. Kotrschal A, Rogell B, Bundsen A, Svensson B, Zajitschek S, Brannstrom I, Immler S, Maklakov AA, Kolm N: Artificial selection on relative brain size in the guppy reveals costs and benefits of evolving a larger brain. Curr Biol 2013, 23:168-171.

22. Snell-Rood EC, Davidowitz G, Papaj DR: Reproductive tradeoffs of learning in a butterfly. Behavioral Ecology 2011, 22:291-302.

23. Chittka L, Niven J: Are bigger brains better? Curr Biol 2009, 19:R995-R1008.

24. Christiansen IC, Szin S, Schausberger P: Benefit-cost trade-offs of early learning in foraging predatory mites Amblyseius swirskii. Sci Rep 2016, 6:23571.

25. Burger JMS, Kolss M, Pont J, Kawecki TJ: Learning ability and longevity: a symmetrical evolutionary trade-off in Drosophila. Evolution 2008, 62:1294-1304.

26. Mery F, Kawecki TJ: A fitness cost of learning ability in Drosophila melanogaster. Proceedings of the Royal Society of London Series B-Biological Sciences 2003, 270:2465-2469. 
27. Alghamdi A, Dalton L, Phillis A, Rosato E, Mallon EB: Immune response impairs learning in free-flying bumble-bees. Biol Lett 2008, 4:479-481.

28. Gegear RJ, Otterstatter MC, Thomson JD: Bumble-bee foragers infected by a gut parasite have an impaired ability to utilize floral information. Proceedings of the Royal Society B-Biological Sciences 2006, 273:1073-1078.

29. Iqbal J, Mueller U: Virus infection causes specific learning deficits in honeybee foragers. Proc Biol Sci 2007, 274:1517-1521.

30. Jaumann S, Scudelari R, Naug D: Energetic cost of learning and memory can cause cognitive impairment in honeybees. Biol Lett 2013, 9:20130149.

31. Placais P-Y, Preat T: To favor survival under food shortage, the brain disables costly memory. Science 2013, 339:440-442.

32. Mery F, Kawecki TJ: A cost of long-term memory in Drosophila. Science 2005, 308:1148.

33. Dukas R: Costs of memory: Ideas and predictions. Journal of Theoretical Biology 1999, 197:41-50.

34. Laughlin SB, de Ruyter van Stevenick RR, Anderson JC: The metabolic cost of neural information. Nature Neuroscience 1998, 1:36-41.

35. Burke CJ, Waddell S: Remembering nutrient quality of sugar in Drosophila. Current Biology 2011, 21:746-750.

36. Fujita M, Tanimura T: Drosophila evaluates and learns the nutritional value of sugars. Curr Biol 2011, 21:751-755.

37. Kikuchi DW, Sherratt TN: Costs of learning and the evolution of mimetic signals. Am Nat 2015, 186:321-332. 
*38. Foucaud J, Moreno C, Pascual M, Rezende EL, Castaneda LE, Gibert P, Mery F:

Introduced Drosophila subobscura populations perform better than native populations during an oviposition choice task due to increased fecundity but similar learning ability. Ecol Evol 2016, 6:1725-1736.

The authors look across multiple populations of flies, testing the general idea that successfully introduced species are more cognitively flexible, and finding that having more offspring can also result in good performance in terms of invasibility. The discussion of the role of learning in adaptive behavior is excellent.

39. Hollis B, Kawecki TJ: Male cognitive performance declines in the absence of sexual selection. Proc Biol Sci 2014, 281:20132873.

40. Hollis KL, Guillette LM: What associative learning in insects tells us about the evolution of learned and fixed behavior. International Journal of Comparative Psychology 2015, 28:1-18.

41. Carrasco D, Larsson MC, Anderson P: Insect host plant selection in complex environments. Current Opinion in Insect Science 2015, 8:1-7.

42. Dukas R: Effects of learning on evolution: robustness, innovation and speciation. Animal Behaviour 2013, 85:1023-1030.

43. Sih, A, Del Giudice, M: Linking behavioural syndromes and cognition: a behavioural ecology perspective. Philosophical Transactions of the Royal Society B. 367:2762-2772.

*44. Croston R, Branch CL, Kozlovsky DY, Dukas R, Pravosudov VV: Heritability and the evolution of cognitive traits. Behavioral Ecology 2015, 26:1447-1459. 
The authors review the current state of work on heritability of cognitive traits across a range of better understood traits. They focus on the connection between genes and environment, and the variation in neural and cognitive traits, and how this responds to selection.

45. Versace E: Experimental evolution, behavior and genetics: associative learning as a case study. Current Zoology 2015, 62:226-241.

*46. Schlotterer C, Kofler R, Versace E, Tobler R, Franssen SU: Combining experimental evolution with next-generation sequencing: a powerful tool to study adaptation from standing genetic variation. Heredity (Edinb) 2015, 114:431-440.

This review covers the use of Pool-seq methods, which feature sequencing samples of many individuals, along with specific analysis techniques, and how the combination of this relatively cost-effective method with experimental evolution can allow for measuring changes in allele frequencies.

47. Versace E, Reisenberger J: Large-scale assessment of olfactory preferences and learning in Drosophila melanogaster: behavioral and genetic components. PeerJ 2015, 3:e1214.

48. Schlotterer C, Tobler R, Kofler R, Nolte V: Sequencing pools of individuals - mining genome-wide polymorphism data without big funding. Nat Rev Genet 2014, 15:749763.

49. Nepoux V, Babin A, Haag C, Kawecki TJ, Le Rouzic A: Quantitative genetics of learning ability and resistance to stress in Drosophila melanogaster. Ecol Evol 2015, 5:543556. 
Figure Legends

Figure 1. We define the certainty of the best action, $p$, as the probability that a given action results in maximized fitness. We define the reliability of experience, $q$, as the conditional probability that a prior experience predicts the best action in a later choice. We imagine individuals can either be guided by experience, or ignore experience and choose with something like an innate preference. A learning organism who is guided by a given experience will choose the correct, or best action, with probability $q$. An organism not learning will simply choose the best action with a probability $p$. The learner will do better as long as $q$ is greater than $p$. See Dunlap \& Stephens 2009 for details.

Figure 2. Experimental preparation, modified from Mery \& Kawecki 2002. Quinine, which is aversive to flies, is paired with a substrate (A) in the experience phase. No quinine is present in the consequence phase, which measures how choice is influenced from the experience with the quinine pairing. If learning has occurred, flies will oviposit on the substrate not previously paired with quinine (substrate B). To select for flies that show behavior consistent with learning, eggs would be collected from substrate B following the consequence phase. The best substrate is simply defined as where the eggs are collected from to rear the next generation. We can manipulate certainty $(p)$, by varying the probability with which the eggs are collected from substrate A. For instance, for certainty of $p=1.0$, all eggs would be reared from orange every generation, while with $p=0.5$, half the generations eggs would be reared from orange, while half are reared from pineapple. We manipulate reliability $(q)$ by how the quinine pairing correctly 
indicates the worst place to lay eggs. With $q=1$, quinine is a perfectly reliable indicator of where not to oviposit; with $q=0.5$, quinine is a correct indicator half of the time.

Figure 3a. Data from Dunlap \& Stephens 2009. Results of learning assays following 30 generations of selections at the most extreme predictions of the model. Flies evolving under high reliability but low certainty show an enhanced ability to learn in comparison to controls and flies evolving under low reliability and high certainty. Each treatment summarizes data from 12 replicate populations. Bars depict 95\% confidence intervals.

Figure 3b. Data from Dunlap \& Stephens 2014. Flies evolving in environments where color, but not odor, was a reliable predictor of the best environment, show enhanced learning to color and not odor (left panel). And vice versa for odor and color (right panel). Replicate populations were assayed following 40 generations of selections. The figure depicts the treatments with the strongest predictions of a four treatment factorial experiment with high and low levels of reliability for each odor and color stimuli. Treatments where both classes of stimuli are unreliable, and when both are completely reliable are not shown. Bars depict standard errors.

Figure 4. Predictions from the Rubi \& Stephens model [19]. The model assumes that two underlying states are possible, good and bad. The state is good with probability $p$. In addition, it assumes that two forms of experience are available $\mathrm{S}$ (which occurs as $\mathrm{S}+$ or $\mathrm{S}-$ ) and $\mathrm{T}$ (which occurs as T+ or T-). The 'plus' forms of S \& T indicate that the underlying state is good with reliabilities $q$ and $r$ respectively. The model assumes that $q>r$. The figure shows how $q$ and $r$ interact to determine the use of the two forms of information. The figure shows five distinct 
regions: A. Always accept ( $p$ very high); B. Accept unless both indicate 'bad'; C. Use the single most reliable form of experience; D. Reject unless both indicate good; and D Always reject ( $p$ very low). 


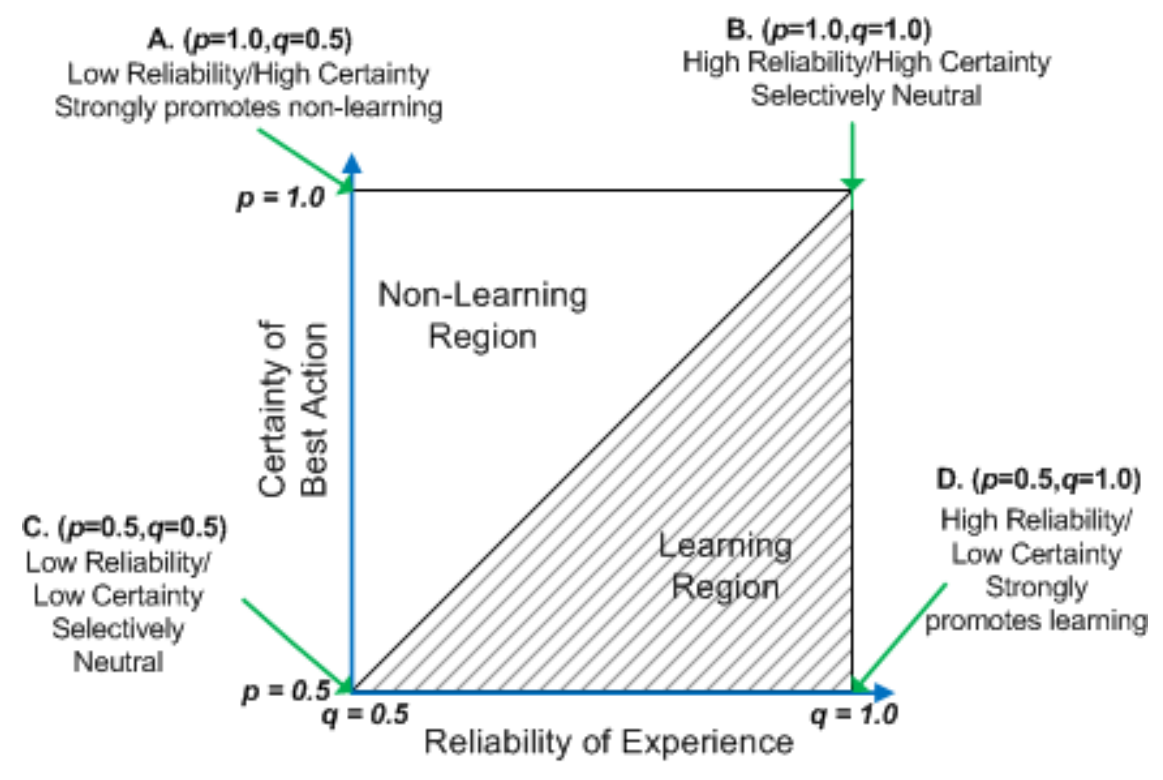


Stage 1: Experience Phase

Flies experience quinine paired with either orange or pineapple

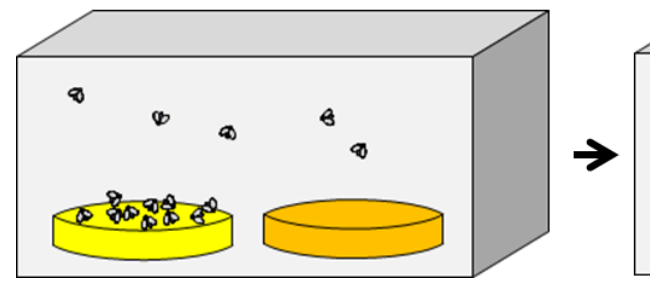

Stage 2: Consequence Phase

No quinine present. Flies can lay eggs on orange or pineapple

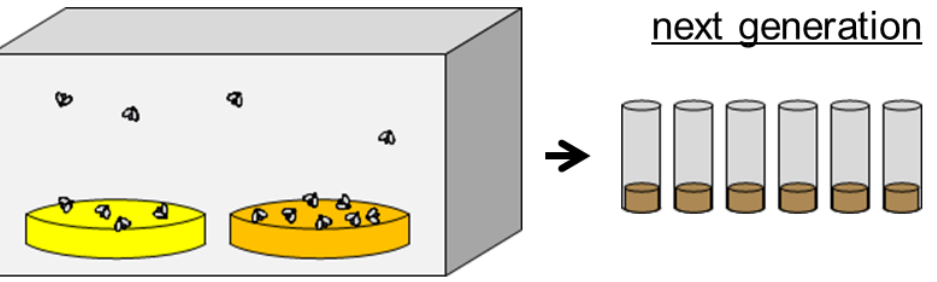



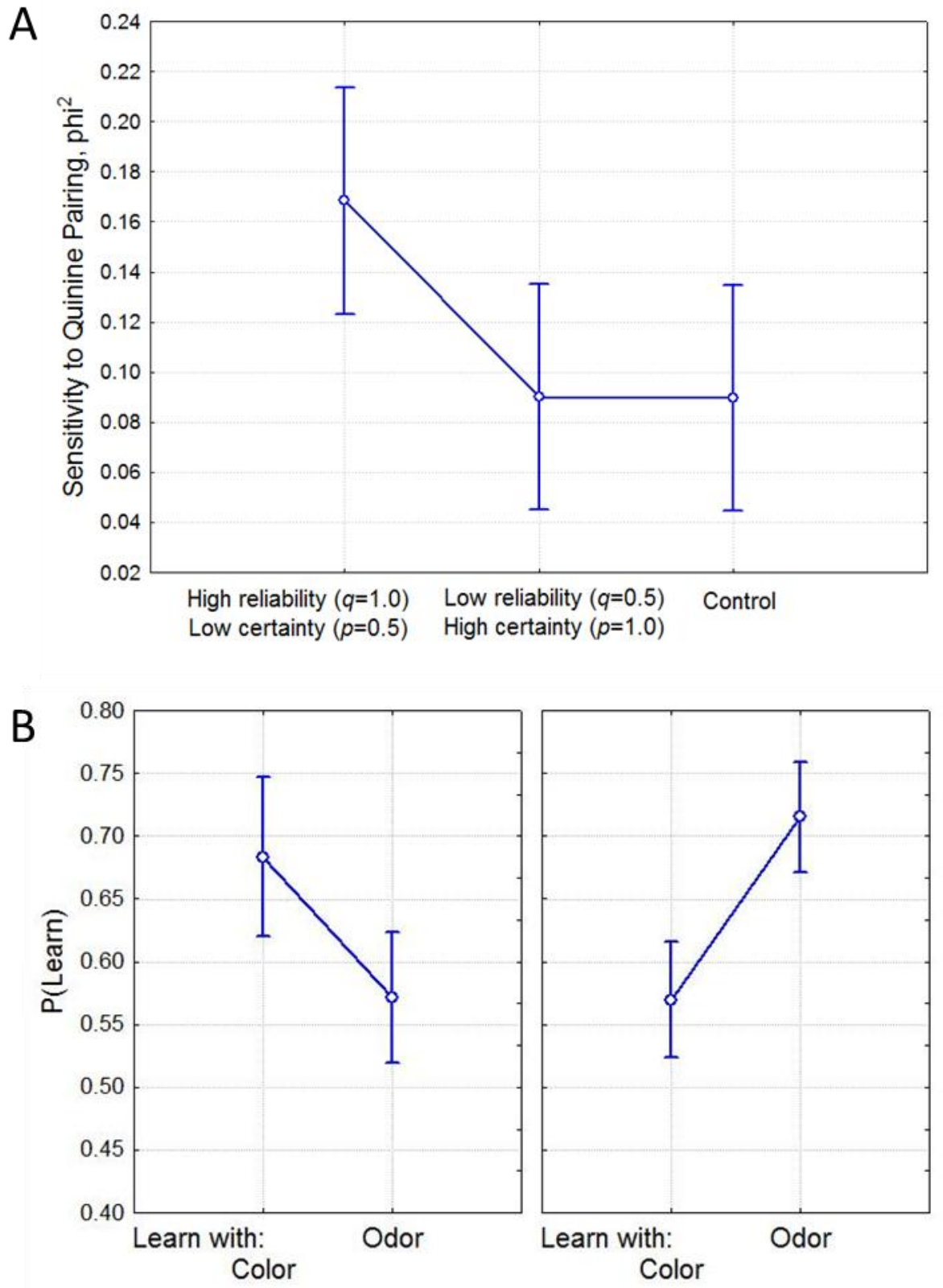


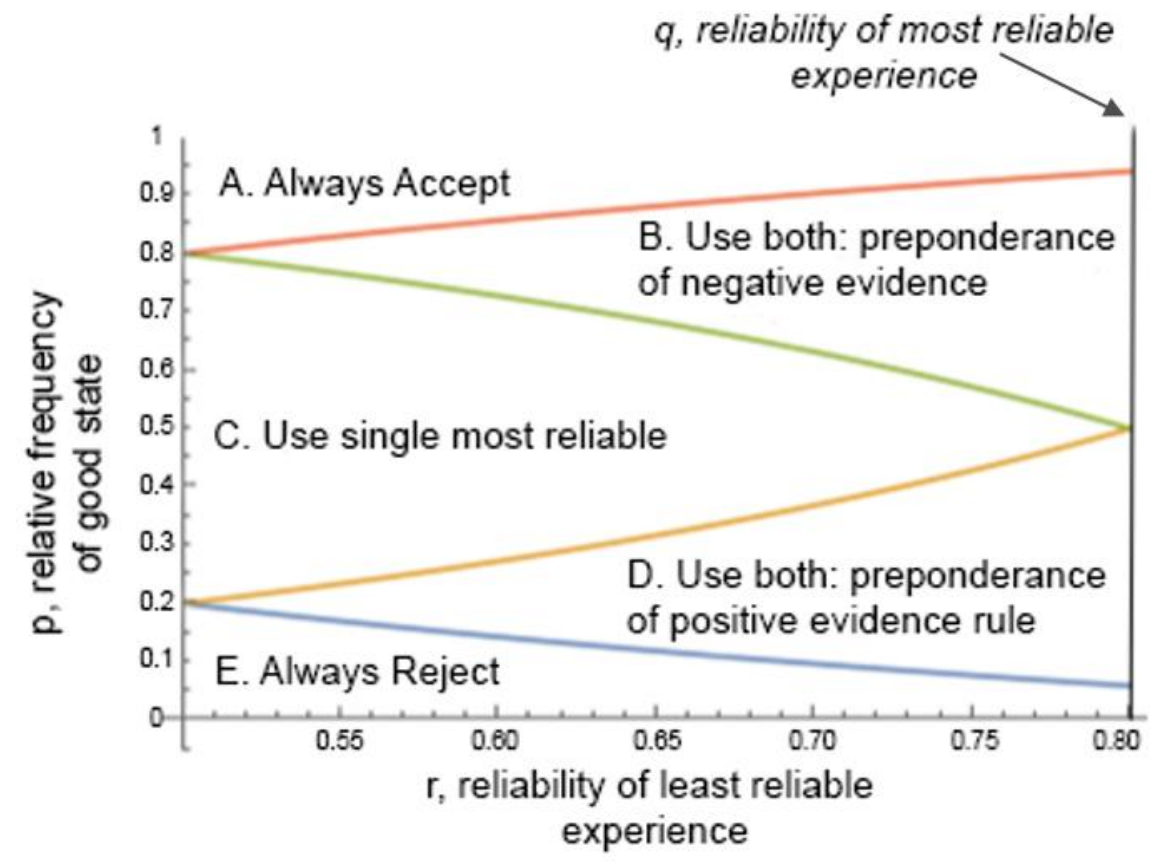

